\title{
Parto Natural. ¿Qué nos dice la evidencia?
}

\author{
Jorge Andrés Carvajal ${ }^{1 *}$, Carolina P. Martinovic ${ }^{1}$, Camila A. Fernández
}

Resumen: Introducción: Es deseable que el proceso del parto concluya con una madre y un recién nacido sano, y sea un momento especial e íntimo. El Parto Natural, ha sido propuesto como una opción para mejorar la satisfacción de las mujeres con el proceso del parto. Sin embargo, no existen definiciones serias respecto a qué es el Parto Natural, introduciendo dificultades en la atención de mujeres que solicitan un Parto Natural. Métodos: El objetivo de este artículo es revisar la mejor evidencia disponible para entender cómo debe definirse un Parto Natural y analizar si se asocia a riesgos mayores que los de la atención médica habitual del parto. Resultados: Hemos comprobado que no existen definiciones científicas consensuadas para precisar qué es el Parto Natural, dejando espacio para discusión sobre su verdadero significado. No existen estudios de diseño aleatorizado y controlado que comparen el resultado materno/perinatal del parto natural comparado con la atención habitual del parto. Las intervenciones médicas, usadas en la atención médica del parto, y que podrían ser evitadas en el Parto Natural, producen algunos cambios favorables y otros deletéreos respecto de la salud materna y perinatal. Conclusiones: La decisión de optar por el Parto Natural debe ser discutida con las mujeres que lo solicitan, precisando con ella y su pareja cuál es su concepto de parto natural o qué es lo que desean incluir o evitar, los riesgos y beneficios asociados a cada una de las intervenciones deben ser expuestos por el equipo médico, para adoptar un plan de manejo individualizado.

Palabras clave: Parto Natural; evidencia científica; riesgo perinatal; riesgo materno; medicalización del parto; plan de parto.

Abstract: Introduction: It is desirable that the birth process concludes with a healthy mother and newborn, while at the same time being a special and intimate moment. Natural childbirth has been proposed as a recent option to improve the satisfaction of women with the process of childbirth. However, there are no serious definitions regarding what is or should be a Natural Childbirth, introducing difficulties in the care of women who request a Natural Birth. Methods: The objective of this article is to review the best available evidence to understand how Natural Childbirth should be defined and to analyze if it is associated with greater maternal or perinatal risks than those of usual medical care at birth. Results: We verified that there are no agreed scientific definition to specify what Natural Childbirth is, leaving space for discussion about its true meaning. There are no randomized, controlled trials comparing the maternal/ perinatal outcome of natural childbirth compared to usual medical care during labor/delivery. Medical interventions used in childbirth care that could be avoided in natural birth produce some favorable changes and some deleterious changes in maternal and perinatal health. Conclusions: The decision to choose Natural Childbirth must be discussed with the women who request it, specifying with her and her partner's concept of natural childbirth or what they wish to include or avoid, the risks and benefits associated with these interventions must be exposed by the medical team, to adopt an individualized management plan.

Keywords: Natural Childbirth; Scientific Evidence; Perinatal Risk; Maternal Risk; Medicalization of childbirth; Birth plan.

Fecha de envío: 30 de diciembre de 2016 - Fecha de aceptación: 30 de abril de 2017

\section{Introducción}

El parto es la culminación del embarazo, el punto final tras una espera de varios meses, que termina con el nacimiento de un nuevo individuo, es el inicio de la vida extrauterina, y es por esto que representa un momento de gran importancia no solo desde el punto de vista médico, sino también psicosocial. En este contexto se mezclan muchos deseos e intereses, entremezclándose por una parte la preocupación de que sea un parto exitoso desde el punto de vista médico, con una madre y un recién nacido sano, y por otra parte, que sea un momento especial e íntimo (Austin \& Leader, 2000).

(1) Unidad de Medicina Materno Fetal. División de Obstetricia y Ginecología. Escuela de Medicina. Facultad de Medicina. Pontificia Universidad Católica de Chile. Santiago. Chile

Autor de correspondencia: jcarva@med.puc.cl 
Es así como últimamente nos hemos visto enfrentados a una nueva corriente en relación al parto, la cual proclama el llamado Parto Natural. Desafortunadamente ninguna organización internacional prestigiosa relacionada con la Obstetricia y Ginecología ha consensuado una definición de lo que debemos entender por Parto Natural. El sistema de búsqueda PubMed (National Center for Biotechnology Information -NCBI- de la National Library of Medicine -NLM) establece como definición de Parto Natural (Natural Childbirth): "parto sin intervención médica, que usualmente involucra técnicas de relajación".

El Parto Natural sería, de acuerdo a páginas de divulgación no científica sobre el tema, una modalidad de nacimiento íntimo, acompañado de la familia y en algunas ocasiones con la participación de doulas; siendo estas últimas mujeres que acompañan y animan a la paciente durante el trabajo de parto para hacerla sentir más contenida y empoderada. Muchas veces se busca que los Partos Naturales ocurran en recintos no hospitalarios, tales como el domicilio o "casas de parto", con el fin de evitar intervenciones médicas frecuentemente utilizadas durante el trabajo de parto tales como el uso de anestesia, ocitocina, o la monitorización fetal continua, y que los defensores del Parto Natural consideran como innecesarias o francamente nocivas (Dietz \& Exton, 2016).

El fundamento psicosocial de los grupos que promocionan el Parto Natural es el favorecimiento de la conexión inicial con el recién nacido, estimulando el apego, la relación madre e hijo y la lactancia materna. Quienes defienden el Parto Natural argumentan que el riesgo de depresión posparto es menor en aquellas mujeres que se someten a esta experiencia (MINSAL, 2008; Dietz \& Exton, 2016).

Producto de una gran promoción mediática, y la presión de los grupos interesados, el deseo de un Parto Natural crece cada día más en nuestro país y representa una consulta habitual en el contexto del control prenatal. Muchas veces incluso, las pacientes, acuden a sus médicos tratantes con un "plan de parto", documento que confeccionan en conjunto con sus parejas o familias y representa sus ideas respecto a lo que desean al momento de la atención del parto.

La solicitud de las parejas muchas veces contrasta con la práctica obstétrica habitual, generando tensión en la relación médico-paciente, pues con frecuencia el médico estima que la mujer se expone a riesgos innecesarios, para ella o su hijo, al solicitar evitar algunas medidas específicas que han demostrado científicamente ser útiles en asegurar bienestary/o que se usan rutinariamente en su centro (Chalmers, 1992; MINSAL, 2008; Olsen \& Clausen, 2012; Dietz \& Exton, 2016).

Esta investigación tiene como objetivo revisar la mejor evidencia disponible respecto del efecto materno y perinatal del parto natural, comparado con la práctica habitual de atención del parto. El conocimiento así generado permitirá a los médicos y sus pacientes tomar decisiones informadas, en base a la mejor evidencia, para garantizar no solo un nacimiento especial y único, sino que también seguro, para la madre y su hijo.

\section{Parto Natural versus Atención Médica del Parto}

La búsqueda en PubMed de investigaciones clínicas referentes a Parto Natural (Natural Childbirth) arroja 44 artículos, solo dos de los cuales corresponden a estudios aleatorizados (Waldenstrom \& Nilsson, 1993, 1994).

Ambos estudios son publicaciones del mismo grupo y reportan el resultado de 1.230 mujeres, con embarazos de bajo riesgo, que fueron aleatorizadas a parto natural (en casas de parto) versus cuidados obstétricos habituales en la maternidad. Las mujeres atendidas en las casas de parto expresaron mayor satisfacción con la atención, especialmente en los aspectos psicológicos de la atención (Waldenstrom \& Nilsson, 1993); sin embargo, reportaron que el parto natural se asoció a mayor dolor comparado con aquel del cuidado obstétrico habitual (Waldenstrom \& Nilsson, 1994).

Estas dos investigaciones tuvieron como objetivo principal evaluar aspectos subjetivos relacionados con la atención del parto, y no reportaron suficientemente potenciales cambios en el resultado materno y perinatal. No existen revisiones sistemáticas que evalúen el impacto clínico del Parto Natural, y las investigaciones clínicas no aleatorizadas disponibles presentan resultados contradictorios respecto a satisfacción, uso de anestesia y otros resultados clínicos de menor relevancia (Eckert et al., 2001; Bloom et al., 2006; Bergstrom et al., 2009).

También es posible obtener información relevante respecto a la seguridad y beneficios del parto natural, si se observan los resultados de atención hospitalaria en recintos especialmente creados para efectuar las mínimas intervenciones posibles durante la atención del parto, llamados escenarios alternativos. Existe una revisión sistemática que midió los efectos de la atención del parto en escenarios hospitalarios alternativos, comparados con la atención en escenarios hospitalarios habituales. Se observó que el uso de escenarios alternativos se asoció a una mayor probabilidad de no uso de analgesia/anestesia intraparto (RR: 1.17; IC 95\% 1.01 - 1.35), parto vaginal espontáneo (RR 1.04; IC 95\% 1.02 - 1.06), amamantamiento a las 6-8 semanas posparto (RR 1.04; IC 95\% 1.02 - 1.06), y visión muy positiva del proceso (RR 1.96; IC 95\% 1.78 - 2.15). El uso de estos escenarios alternativos se asocia a reducción del uso de epidural (RR 0.82; IC 95\% 0.75 - 0.89), aceleración ocitócica (RR 0.78 ; IC 95\% 0.66 - 0.91) y episiotomía (RR 0.83; IC 95\% 0.77 - 0.90). 
No se observaron diferencias en las complicaciones maternas o perinatales en estos dos escenarios. Los autores concluyen que los escenarios alternativos permiten reducir el número de intervenciones médicas, sin incrementar el riesgo materno/perinatal y mejorando la satisfacción materna. Por las características de las investigaciones incluidas, es imposible descartar que en parte estas diferencias se deban a cambios en los médicos y personal que atiende en los dos escenarios (Hodnett et al., 2012).

Ante la falta de estudios de buen diseño que comparen los resultados maternos y perinatales del Parto Natural, respecto a la atención médica habitual del parto, revisaremos el impacto de cada una de las intervenciones involucradas en la atención habitual del parto y que son llamadas a evitarse en un parto natural; en base a los datos aportados por revisiones sistemáticas.

\section{Parto vaginal versus cesárea}

Uno de los objetivos básicos del parto natural es lograr un parto vaginal, el cual ha demostrado múltiples beneficios, tanto para la madre como para el recién nacido, comparado con la operación cesárea (Villar et al., 2006). Desafortunadamente, debemos comentar, más allá de los objetivos de esta revisión, que Chile presenta una alta tasa de cesáreas; un tema que debe ser analizado por todos los grupos médicos y del gobierno.

En cuanto a riesgo neonatal, el parto vaginal, en comparación con la cesárea, se asocia a menor morbilidad respiratoria, sin diferencias significativas en cuanto a fracturas traumáticas del recién nacido, parálisis braquial congénita, muerte neonatal y apgar bajo. El parto vaginal tiene riesgo de distocia de hombro, entre $0,2-2 \%$ de los casos, riesgo que no existe en la operación cesárea (Gregory et al., 2012). (Tabla 1).

Tabla 1: Riesgos neonatales y maternos del parto vaginal versus cesárea.

\begin{tabular}{|c|c|c|c|}
\hline \multicolumn{4}{|c|}{ Riesgos Neonatales } \\
\hline & & Parto Vaginal & Cesárea \\
\hline Laceraciones & & --- & $1-2 \%$ \\
\hline \multirow[t]{3}{*}{ Trauma obstétrico } & Distocia hombro & $0,2-2,0 \%$ & --- \\
\hline & Parálisis braquial congénita & $2-5 / 1.000$ & $2,1 / 1.000$ \\
\hline & Fracturas & $1-2 \%$ & $1-2 \%$ \\
\hline Adaptación & Morbilidad respiratoria (término) & $<1 \%$ & $1-4 \%$ \\
\hline \multirow[t]{2}{*}{ Resultado } & Muerte & Sin diferencia & Sin diferencia \\
\hline & Apgar bajo & Sin diferencia & Sin diferencia \\
\hline \multicolumn{4}{|c|}{ Riesgos Maternos } \\
\hline \multirow[t]{5}{*}{ Quirúrgicas } & Morbilidad severa & $0.9 \%$ & $2.7 \%$ \\
\hline & Hemorragia post parto & $1-5 \%$ & $1-8 \%$ \\
\hline & Histerectomía & $2-5 / 1000$ & $10-20$ veces \\
\hline & Embolía líquido amniótico & $3.3-7.7 / 100000$ & $15.8 / 100000$ \\
\hline & Muerte materna & $3.6 / 100000$ & $13.3 / 100000$ \\
\hline \multirow[t]{2}{*}{ Anestesia } & Regional & $0.06 / 1000$ & $0.13-0.29 / 1000$ \\
\hline & General & --- & 2 veces \\
\hline \multirow[t]{3}{*}{ Trauma Perineal } & Desgarros $3^{\circ}-4^{\circ}$ grado & $2-5 \%$ & --- \\
\hline & Incontinencia urinaria & $21-32 \%$ & $0-10 \%$ \\
\hline & Incontinencia fecal & $10-20 \%$ & $3 \%$ \\
\hline \multirow[t]{2}{*}{ Infeccioso } & Fiebre & $1-2 \%$ & $2.5 \%$ \\
\hline & Infección herida operatoria & $0.05 \%$ & $3 \%$ \\
\hline \multirow[t]{4}{*}{ Próximo embarazo } & Rotura uterina & --- & $1 \%$ \\
\hline & DPPNI & $0.74 \%$ & $0.95 \%$ \\
\hline & Placenta previa & $0.38 \%$ & $1,5-3,0$ veces \\
\hline & Acretismo & --- & $1-40 \%$ \\
\hline \multirow[t]{2}{*}{ Reproductivos } & Infertilidad & --- & Aumenta riesgo \\
\hline & Mortinato & --- & Aumenta riesgo \\
\hline Apego & Lactancia & --- & Disminuye \\
\hline
\end{tabular}

DPPNI = desprendimiento prematuro de placenta normoinserta (Bauer \& Bonanno, 2009; Gregory et al., 2012) 
Por otra parte, cada vez existe más información acerca de las diferencias en la microbiota de niños nacidos por parto vaginal versus cesárea, lo cual parece jugar un papel fundamental en el desarrollo del sistema inmunológico del recién nacido. De hecho, existe asociación entre vía del parto cesárea y mayor riesgo de alergias alimentarias, rinitis alérgica, asma, enfermedad de Crohn y diabetes mellitus tipo I (Bager et al., 2008; Cardwell et al., 2008; Thavagnanam et al., 2008; Li et al., 2014).

En cuanto al riesgo materno a corto plazo, el parto vaginal respecto de la cesárea ha demostrado menores tasas de: morbilidad severa, hemorragia posparto, histerectomía obstétrica, embolia de líquido amniótico y muerte materna. Sin embargo, el parto vaginal tiene mayor riesgo de trauma perineal; desgarros de 3-4 grado, incontinencia urinaria e incontinencia fecal comparado con la cesárea (Gregory et al., 2012); como se observa en la Tabla 2. Las complicaciones de la anestesia también son más frecuentes en el parto por cesárea que en el parto vaginal (Gregory et al., 2012) (Tabla 1).

En relación a los riesgos a mediano y largo plazo asociados a la vía del parto, pacientes con cesárea tienen mayor riesgo de infección de la herida operatoria (Gregory et al., 2012) y en un embarazo siguiente mayor probabilidad de rotura uterina, placenta previa y acretismo placentario, comparado con mujeres que han tenido un parto vaginal (Bauer \& Bonanno, 2009; Gregory et al., 2012). (Tabla 1). Las mujeres cuyo primer parto se resolvió por cesárea, tiene un riesgo mayor de infertilidad conyugal y de muerte fetal in utero (Gregory et al., 2012) (Tabla 1).

\section{Manejo activo del trabajo de parto}

Se refiere a un conjunto de acciones que tienen como objetivo acortar el trabajo de parto, y que consiste en: amniotomía precoz, vigilancia de la progresión de la dilatación cervical y aceleración ocitócica en fase activa. El manejo activo del trabajo de parto reduciría la duración de la fase activa, lográndose el parto en el $63 \%$ de las paciente a las $6 \mathrm{~h}$ y en el $98 \%$, a las $12 \mathrm{~h}$.

El manejo activo del trabajo de parto, comparado con el manejo expectante (sin amniotomía ni aceleración ocitócica rutinaria) no se asocia a cambios en la incidencia de cesárea, estado fetal no tranquilizador, desprendimiento prematuro de placenta normoinserta, distocia de hombro, desgarro perineal, insatisfacción con la experiencia del parto (Pates \& Satin, 2005).

\section{Aceleración ocitócica}

Consiste en la administración de ocitocina endovenosa a aquellas pacientes en trabajo de parto activo, con el objetivo de acelerar la progresión del trabajo de parto. El uso de aceleración ocitócica rutinaria aumenta significativamente: la probabilidad de parto vaginal (RR 1.09; IC 95\% 1.03 - 1.17), el riesgo de taquisistolia (RR 2.9; IC $95 \% 1.21$ - 6.94) y el dolor asociado a las contracciones uterinas (100\% vs 2,5\%; $\mathrm{p}<0.01$ ); además, la aceleración ocitócica reduce el uso de antibióticos (RR 0.45; IC 95\% 0.21 - 0.99), probablemente asociado a la disminución en los tiempos del trabajo de parto. El uso de aceleración ocitócica rutinaria no se asocia a cambios en el resultado perinatal (Wei et al., 2009).

\section{Amniotomía rutinaria precoz}

La rotura artificial de membranas es utilizada frecuentemente como una manera de evaluar las características del líquido amniótico, constituyendo un dato importante en la evaluación del bienestar fetal, especialmente cuando nos vemos enfrentados a alteraciones en la cardiotocografía.

La rotura artificial de membranas ovulares, efectuada de modo rutinario y precoz, con el objetivo de acortar el tiempo del trabajo de parto, permitiría el descenso de la cabeza fetal y la liberación de prostaglandinas a nivel cervical, lo que aumentaría la dinámica uterina. Sin embargo, la rotura artificial de membranas ovulares rutinaria y precoz no muestra diferencias significativas en duración de la primera fase del trabajo de parto (diferencia promedio-20.4 min), tasa de cesárea; (RR 1.27; IC 95\% 0.99 - 1.62), satisfacción materna, ni apgar bajo (RR 0.57; IC 95\% 0.31 - 1.06) (Smyth et al., 2013).

\section{Amniotomía y aceleración ocitócica para el tratamiento de fase de dilatación prolongada}

La velocidad de progresión del trabajo de parto debe ser vigilada y comparada con patrones de normalidad: partograma. Esta vigilancia permite determinar qué mujeres no siguen un curso fisiológico en su trabajo de parto, permitiendo adoptar medidas correctivas cuando sea necesario.

La combinación de amniotomía y/o aceleración ocitócica en mujeres cuya fase de dilatación progresa más lento que lo habitual (de acuerdo a las curvas de partograma) se ha asociado a una reducción en la tasa de cesárea (RR 0.87; IC 95\% 0.77 - 0.99) y un acortamiento en la duración del trabajo de parto (diferencia media - 1.28 h; IC 95\% - 1.97 a -0.59 h), sin cambios en morbilidad materna o fetal (Wei et al., 2013).

\section{Inducción del trabajo de parto comparado con evolución espontánea}

Si un embarazo progresa de modo fisiológico, es usual esperar el inicio espontáneo del trabajo de parto hasta las 41 o 42 semanas, pues después de eso se ha demostrado mayor morbimortalidad perinatal (Crowley, 2000; Sanchez-Ramos et al., 2003; Bulletins-Obstetrics, 2004). 
Una política de inducción del trabajo de parto en embarazos mayores o iguales a 39 semanas, comparado con manejo expectante, reduce significativamente la pérdida de sangre (diferencia media $-57.59 \mathrm{ml}$; IC 95\% -83.96 a -31.21 ml), la frecuencia de meconio (RR 0.32 ; IC $95 \% 0.18$ - 0.57) y el peso fetal (diferencia media - $135.51 \mathrm{~g}$; IC 95\% [-205.24 a -65.77 g) (Saccone \& Berghella, 2015). Esta opción de inducción rutinaria no se asocia a cambios significativos en la tasa de cesárea, uso de fórceps, frecuencia de corioamnionitis o resultado perinatal (Saccone \& Berghella, 2015).

\section{Anestesia Epidural}

El dolor abdominal y perineal durante el trabajo de parto puede ser manejado con medidas farmacológicas o no farmacológicas. Entre las medidas no farmacológicas se cuentan inmersión en agua, relajación, acupuntura y masajes. Dentro de las medidas farmacológicas se incluyen la inhalación de óxido nitroso, opiáceos endovenosos y analgesia regional de tipo epidural; constituyendo esta última la técnica más ampliamente utilizada en nuestro medio.

No existen revisiones sistemáticas que comparen el alivio del dolor usando técnicas no farmacológicas versus farmacológicas. Las investigaciones clínicas reportan datos contradictorios respecto a la necesidad de analgesia epidural en usuarias de medidas no farmacológicas.

Al comparar el uso de epidural versus opiáceos endovenosos, se observa que la analgesia epidural presenta mejor eficacia en el manejo del dolor; sin embargo, también presenta mayor riesgo de parto vaginal asistido, hipotensión, fiebre, retención urinaria posparto, prolongación del expulsivo y mayor necesidad de aceleración ocitócica (Anim-Somuah et al., 2011). Como se observa en la tabla 2, el uso de analgesia epidural no se asocia a aumento en la tasa de cesárea (Anim-Somuah et al., 2011).

Tabla 2: Comparación de riesgos maternos perinatales relacionados con la analgesia epidural comparado con analgesia endovenosa con opiáceos

\begin{tabular}{|l|c|c|}
\hline & RR o DM & IC 95\% \\
\hline Aumenta & 1.42 & {$[1.28-1.57]$} \\
\hline Riesgo de parto vaginal asistido & 18.23 & {$[5.09-65.35]$} \\
\hline Hipotensión materna & 17.05 & {$[4.82-60.39]$} \\
\hline Retención urinaria & 3.34 & {$[2.63-4.23]$} \\
\hline Fiebre materna & 1.19 & {$[1.03-1.39]$} \\
\hline Mayor necesidad de ocitocina & DM 13.66 min & {$[6.67-20.66]$} \\
\hline Prolongación del expulsivo & DM -3.36 & {$[5.41-1.31]$} \\
\hline Disminuye & 1.1 & {$[0.97-1.25]$} \\
\hline Dolor (escala liker) &
\end{tabular}

RR: Riesgo Relativo, IC 95\%: Intervalo de confianza del 95\%, DM: Diferencia Media (Anim-Somuah et al., 2011)

\section{Episiotomía restrictiva versus rutinaria}

La episiotomía rutinaria fue propuesta como el método para evitar los desgarros vagino-perineales graves (3-4 grado). Sin embargo, esta recomendación provino de observaciones no sistemáticas y de opiniones de expertos no bien avaladas por evidencia científica.

Los estudios randomizados, efectuados a fines del siglo pasado, compararon grupos randomizados a episiotomía rutinaria versus episiotomía restrictiva, esta última efectuada cuando el tratante juzgara, en base a su experiencia, que la episiotomía era indispensable; en esas condiciones, los grupos con episiotomía rutinaria resultaron en efectuar la episiotomía al $75 \%$ de las mujeres versus al 25\% de aquellas en el grupo restrictivo (Carroli \& Mignini, 2009).

El uso restrictivo de la episiotomía, comparado con episiotomía de rutina, presenta menores tasas de trauma perineal severo, menor necesidad de uso de suturas y menor tasa de complicaciones de la herida; sin diferencia en la frecuencia de dispareunia, incontinencia urinaria o dolor (Carroli \& Mignini, 2009). (Tabla 3).

Tabla 3: Riesgos relacionados con práctica de episiotomía restrictiva versus rutinaria

\begin{tabular}{|l|c|c|}
\hline \multicolumn{2}{|l|}{ RR } & IC 95\% \\
\hline Aumenta & 1.84 & {$[1.61-2.1]$} \\
\hline Trauma perineal anterior & \multicolumn{2}{|l|}{} \\
\hline Disminuye & 0.67 & {$[0.49-0.91]$} \\
\hline Trauma perineal severo & 0.71 & {$[0.61-0.81]$} \\
\hline Uso de suturas & 0.69 & {$[0.56-0.85]$} \\
\hline Complicaciones de la herida & & \\
\hline Sin cambios & 1.02 & {$[0.90-1.16]$} \\
\hline Dispareunia & 0.98 & {$[0.79-1.20]$} \\
\hline Incontinencia urinaria & 1.00 & {$[0.78-1.27]$} \\
\hline Dolor perineal &
\end{tabular}

RR: Riesgo Relativo, IC 95\%: Intervalo de confianza del 95\% (Carroli \& Mignini, 2009)

\section{Monitorización de los latidos cardíacos fetales durante el trabajo de parto}

La vigilancia de los latidos cardíacos fetales durante el trabajo de parto reduce la tasa de muerte perinatal desde 70/1.000 a 4/1.000 nacidos, esto a costa de un aumento en la tasa de cesárea del 3,5\% al $18 \%$. Esta reducción muy significativa del riesgo de muerte fetal intraparto justifica que en la actualidad todas las recomendaciones internacionales consideran imprescindible la vigilancia de los latidos cardíacos fetales durante el trabajo de parto (Chez et al., 2000).

La vigilancia de los latidos cardíacos fetales puede ser realizada de modo intermitente o continuo (cardiotocografía). Al comparar ambas técnicas, se observa que la cardiotocografía se asocia a un aumento significativo en la tasa de cesárea y parto instrumental 
(Tabla 4). La cardiotocografía, comparada con la auscultación intermitente de los latidos cardíacos fetales, se asocia a una reducción significativa en la tasa de convulsiones neonatales, sin diferencias en la frecuencia de: acidosis fetal, necesidad de hospitalización del recién nacido, encefalopatía hipóxico-isquémica, muerte perinatal, secuela neurológica y parálisis cerebral (Chez et al., 2000; Alfirevic et al., 2006). (Tabla 4).

Tabla 4: Comparación de los riesgos relacionados cardiotocografía continua vs auscultación intermitente de los latidos cardíacos fetales durante el trabajo de parto

\begin{tabular}{|l|l|l|}
\hline \multicolumn{2}{|l|}{ RR } & IC 95\% \\
\hline Aumenta & 1.63 & {$[1.29-2.07]$} \\
\hline Tasa de Cesárea & 1.15 & {$[1.01-1.33]$} \\
\hline Parto Instrumental & \multicolumn{2}{|l|}{} \\
\hline Disminuye & {$[0.31-0.80]$} \\
\hline Convulsiones neonatales & 0,50 & {$[0.27-3.11]$} \\
\hline Sin Cambios & 0.92 & {$[0.86-1.18]$} \\
\hline Acidosis fetal & 1.01 & {$[0.04-5.03]$} \\
\hline Hospitalización RN hipóxico- & 0.46 & {$[0.59-1.23]$} \\
\hline $\begin{array}{l}\text { Encefalopatía } \\
\text { isquémica }\end{array}$ & 0.86 & {$[0.83-18.1]$} \\
\hline Muerte perinatal & 3.88 & {$[0.84-3.36]$} \\
\hline $\begin{array}{l}\text { Secuela neurológica 12 meses } \\
\text { vida }\end{array}$ & 1.75 & \\
\hline Parálisis cerebral &
\end{tabular}

RR: Riesgo Relativo, IC 95\%: Intervalo de confianza del 95\% (Chez et al., 2000; Alfirevic et al., 2006)

\section{Parto Domiciliario}

La atención del parto en el domicilio de la mujer, sin atención médica, fue la práctica habitual por siglos; mismo período en que las tasas de morbilidad y mortalidad materna y perinatal eran muy altas. La atención médica del parto llevó a que el evento se produjera en los hospitales, en busca de mayor seguridad. Sin embargo, hoy en día, algunas mujeres desean optar por volver al parto domiciliario. En los países y sistemas de salud donde esta opción está normada y regulada, el parto en el domicilio está reservado solo para mujeres/ embarazos de bajo riesgo.

Los estudios observacionales han sugerido que para multíparas, el parto domiciliario reporta menores intervenciones, y no se asocia a alteraciones del resultado perinatal; mientras que para primíparas el parto domiciliario resultaría en menores intervenciones, pero a expensas de un peor resultado perinatal (OR 1.75, IC 95\% 1.07-2.86; para mal resultado perinatal en parto domiciliario comparado con hospitalario) (Birthplace in England Collaborative et al., 2011). Es importante notar que la gran mayoría de los estudios observacionales reporta el resultado perinatal, pero no el resultado materno.
Solo dos estudios randomizados, controlados, evalúan la seguridad del parto en el domicilio, comparado con el parto en el hospital; sus resultados fueron compilados en una revisión sistemática (Olsen \& Clausen, 2012). La conclusión de esta revisión es que el bajísimo número de mujeres incluidas (11 mujeres) impide obtener conclusiones confiables.

Un problema frecuente al analizar los riesgos del parto domiciliario es que el registro de estadísticas vitales es insuficiente para saber qué pacientes cuya intención primaria era el parto domiciliario son finalmente trasladadas a la maternidad para el parto, aumentando aparentemente el riesgo de aquellos partos ocurridos en el hospital. Un reciente estudio de cohorte retrospectivo evaluó todos los partos ocurridos en Oregon entre 2012 y 2013, luego de mejorar su sistema de registro, permitiendo comparar el grupo de mujeres que habían planeado su parto en el domicilio, respecto de aquellas que planearon tenerlo en el hospital. Se observó que el grupo de parto domiciliario presentó un aumento en la tasa de muerte perinatal, la tasa de convulsiones neonatales y la necesidad de transfusiones maternas, con una disminución en tasa de parto vaginal asistido, tasa de cesárea, así como de inducción y aceleración del trabajo de parto (Snowden et al., 2015). (Tabla 5).

Tabla 5: Riesgos de la atención del parto domiciliario vs atención hospitalaria.

\begin{tabular}{|c|c|c|c|}
\hline & Valores & OR & IC 95\% \\
\hline \multicolumn{4}{|l|}{ Aumenta } \\
\hline Muerte perinatal & $\begin{array}{c}3,9 / 1.000 \text { vs } \\
1,8 / 1.000\end{array}$ & 2.43 & {$[1,37-4,30]$} \\
\hline Convulsiones & $\begin{array}{c}1,3 / 1.000 \text { vs } \\
0,4 / 1.000\end{array}$ & 3.6 & {$[1.36-9.50]$} \\
\hline APGAR $<7,5 \mathrm{~min}$ & & 1.31 & {$[1.04-1.66]$} \\
\hline $\begin{array}{l}\text { Transfusiones } \\
\text { Materna }\end{array}$ & $0,6 \%$ vs $0,4 \%$ & 1.91 & [1.25 - 2.93] \\
\hline \multicolumn{4}{|l|}{ Disminuye } \\
\hline $\begin{array}{l}\text { Parto Vaginal } \\
\text { asistido }\end{array}$ & $1,0 \%$ vs $3,0 \%$ & 0,24 & {$[0.17-0.34]$} \\
\hline Cesárea & $5,3 \%$ vs $23,4 \%$ & 0.18 & {$[0.16-0.22]$} \\
\hline $\begin{array}{l}\text { Inducción del } \\
\text { trabajo de parto }\end{array}$ & & 0.11 & [0.09-0.12] \\
\hline $\begin{array}{l}\text { Aceleración del } \\
\text { trabajo de parto }\end{array}$ & & 0.21 & {$[0.19-0.24]$} \\
\hline
\end{tabular}

OR: Odds Ratio; IC 98\%: Intervalo de confianza del 95\% (Olsen \& Clausen, 2012; Snowden et al., 2015)

\section{Participación de Doulas en el trabajo de parto}

Las Doulas son mujeres que no pertenecen al ámbito médico, cuyo rol es brindar apoyo emocional y/o físico durante el trabajo de parto, y el período posparto, a las mujeres y sus parejas. En nuestros días, en Chile, las Doulas se han transformado en un servicio muchas veces remunerado que ofrece este tipo de acompañamiento, pago que no es cubierto por los sistemas de salud. 
Existen dos estudios clínicos randomizados que comparan los efectos de la presencia o ausencia de la Doula durante el trabajo de parto. Estos estudios mostraron que la participación de la Doula reduce el uso de analgesia (54.4\% vs $66.1 \%$; $\quad$ p < 0.05 , estimula la relación madre-hijo, mejora la expectativa positiva prenatal y autoestima, así como la experiencia como mujer (58.0\% vs. $43.7 \%$; $\mathrm{p}<0.05)$ y la percepción de su cuerpo (58.0\% vs $41.0 \%$; $<<0.05$ ). La presencia de la Doula no reduce la tasa de cesárea, el parto instrumental, la aceleración ocitócica ni la depresión posparto (Gordon et al., 1999; Campbell et al., 2007).

\section{Parto Vertical}

El parto vertical es aquel que ocurre con la mujer en una posición de 45 a 90 grados con respecto al plano horizontal, pudiendo ser de pie o en cuclillas. Los defensores del parto vertical argumentan que facilita el pujo, y es la posición natural por excelencia para el parto; en efecto, algunas etnias (ej. Mapuches), prefieren esta posición para el parto.

Existe una revisión sistemática que evaluó la posición durante el expulsivo, comparando las posiciones verticales respecto a la posición supina o litotomía. El expulsivo en posición vertical se asoció a menor duración del expulsivo (diferencia media - 4.29 m; IC 95\% - 2.95 a -5,64 m), y a una disminución significativa en la tasa de parto vaginal asistido (RR 0.84; IC 95\% 0.73 - 0.98), necesidad de episiotomía (RR 0.84; IC 95\% 0.79 - 0.91), dolor severo durante el expulsivo (RR 0.73; IC 95\% 0.60 - 0.90) y patrones de frecuencia cardíaca fetal anormal (RR 0.31; IC 95\% 0.08 - 0.98). Por otra parte, el expulsivo en posición vertical se asoció a un aumento la frecuencia de sangrado mayor a 500cc (RR 1.68; IC 95\% 1.32 - 2.15) y en la frecuencia de desgarros perineales de segundo grado (RR 1.23; IC 95\% 1.09 - 1.39) (Gupta \& Hofmeyr, 2004; Gupta et al., 2012).

\section{Inmersión en Agua}

Se ha sugerido que la inmersión en tinas calientes durante el período de dilatación o expulsivo puede resultar reconfortante para la madre, y acercarse al modelo del Parto Natural. Una revisión sistemática analizó el efecto de la inmersión en agua durante el trabajo de parto y observó que su uso durante el período de dilatación se asoció a menor uso de anestesia (RR 0.82; IC 95\% 0.70 - 0.98). La inmersión en agua durante el trabajo de parto no produjo cambios significativos en tasa de cesáreas, tasa de fórceps, incidencia de desgarros perineales. Del mismo modo, el resultado perinatal no se vio afectado por la inmersión en agua (Cluett et al., 2004).

Tabla 6: Riesgos materno/perinatales relacionados con el manejo activo o infusión de ocitocina versus manejo expectante del alumbramiento

\begin{tabular}{|c|c|c|}
\hline \multicolumn{3}{|c|}{ Manejo activo del alumbramiento } \\
\hline & $\mathbf{R R}$ & IC 95\% \\
\hline \multicolumn{3}{|l|}{ Reduce } \\
\hline Anemia severa $(\mathrm{Hb}<9 \mathrm{gr} / \mathrm{dl})$ & 0.50 & {$[0.30-0.83]$} \\
\hline Transfusión hemoderivados & 0.35 & {$[0.22-0.55]$} \\
\hline Uso terapéutico uterotónico & 0.19 & {$[0.15-0.23]$} \\
\hline Sangrado mayor a $1000 \mathrm{ml}$ & 0.34 & {$[0.14-0.87]$} \\
\hline \multicolumn{3}{|l|}{ Aumenta } \\
\hline Presión arterial diastólica postparto & 4.10 & {$[1.63-10.3]$} \\
\hline Frecuencia vómitos & 2.47 & {$[1.36-4.48]$} \\
\hline Necesidad analgésicos & 2.53 & {$[1.34-4.78]$} \\
\hline \multicolumn{3}{|l|}{ Sin Cambios } \\
\hline Admisión a UCI neonatal & 0.81 & {$[0.60-1.11]$} \\
\hline Hiperbilirrubinemia de RN & 0.96 & {$[0.55-1.68]$} \\
\hline Lactancia materna al alta & 1.01 & {$[0.96-1.07]$} \\
\hline \multicolumn{3}{|c|}{ Infusión de ocitocina rutinaria para el alumbramiento } \\
\hline \multicolumn{3}{|l|}{ Reduce } \\
\hline Sangrado mayor a $500 \mathrm{ml}$ & 0.53 & {$[0.38-0.74]$} \\
\hline Sangrado mayor a $1000 \mathrm{ml}$ & 0.62 & {$[0.44-0.87]$} \\
\hline Uso terapéutico uterotónico & 0.56 & {$[0.36-0.87]$} \\
\hline \multicolumn{3}{|l|}{ Sin cambios } \\
\hline Anemia severa $(\mathrm{Hb}<9 \mathrm{gr} / \mathrm{dl})$ & 0.80 & {$[0.60-1.00]$} \\
\hline Transfusión hemoderivados & 0.89 & {$[0.44-1.78]$} \\
\hline Duración del alumbramiento $>30 \mathrm{~min}$ & 2.55 & {$[0.88-7.44]$} \\
\hline Remoción manual de la placenta & 1.26 & {$[0.88-1.81]$} \\
\hline Frecuencia vómitos & 0.29 & {$[0.01-6.74]$} \\
\hline
\end{tabular}

RR: Riesgo Relativo, IC 95\%: Intervalo de confianza del 95\% (Begley et al., 2015) 


\section{Manejo activo versus manejo expectante del alumbramiento}

Quienes promueven el Parto Natural proponen que el uso rutinario de ocitocina posparto resulta deletéreo para el apego y la lactancia, además de riesgoso para la salud emocional de la madre, sin contar para esta sugerencia con evidencia científica.

El manejo expectante del alumbramiento implica esperar la aparición de signos de desprendimiento de la placenta, diferir la ligadura del cordón umbilical, y esperar la salida espontánea de la placenta. Por el contrario, el manejo activo del alumbramiento implica: uso rutinario de ocitocina, ligadura precoz del cordón y tracción controlada de la placenta.

La más reciente revisión sistemática mostró que para población general de mujeres, el manejo activo del alumbramiento reduce el riesgo de hemorragia posparto (> $500 \mathrm{o}>1.000 \mathrm{ml}$ ), anemia severa posparto (hemoglobina $<9 \mathrm{~g} / \mathrm{dl}$ ), transfusión de hemoderivados $\mathrm{y}$ uso terapéutico de uterotónicos (dentro de $24 \mathrm{~h}$ posparto). El manejo activo se asoció a incremento significativo en presión arterial diastólica posparto, frecuencia de vómitos y necesidad de analgésicos. No se reportaron diferencias en admisión a UCI neonatal o hiperbilirrubinemia del RN; y no había datos suficientes para extraer conclusiones sobre mortalidad materna (Begley et al., 2015). (Tabla 6)

La revisión sistemática específica para el uso de ocitocina posparto, descartando los otros dos elementos del manejo activo, mostró que su uso reduce significativamente el riesgo de: hemorragia postparto (> $500 \mathrm{ml}$ ) y la necesidad de uterotónicos terapéuticos. (Westhoff et al., 2013). (Tabla 6)

\section{Discusión}

Hemos comprobado que no existen definiciones científicas consensuadas para precisar qué es el Parto Natural, dejando espacio para que las personas (profesionales de la salud y pacientes) generen sus propias creencias sobre qué es o qué debe ser un Parto Natural. No encontramos estudios de diseño aleatorizado y controlado que comparen el resultado materno/perinatal del parto natural comparado con la atención habitual del parto. Las diferentes intervenciones médicas, usualmente asociadas a la atención médica del parto, y que desean ser evitadas en el Parto Natural, alteran positiva o negativamente el resultado materno/ fetal como se observa en las Tablas 1-6.

Mucha de la información no médica a la que acceden las embarazadas propone al Parto Natural como lo bueno, lo "natural", lo "humanizado" o "no medicalizado", levantando a un enemigo potencial en el acto médico. Sin embargo, lo opuesto a natural, en este escenario, no es lo artificial o antinatural, sino que lo cultural; entendiendo la cultura como la creación de lo humano (Kagawa Singer et al., 2016). El modo en que hoy atendemos el parto es producto de la evolución espontánea del acto médico, incluyendo intervenciones y evitando otras, constituyendo un uso habitual que la gran mayoría acepta sin discusiones (MacDonald, 2011). Parece razonable que discutamos la pertinencia de lo que hacemos hoy en día, y en ese escenario el artículo que presentamos resulta de relevancia (Lothian, 2000).

Ante la falta de estudios de buen diseño que comparen los resultados maternos y perinatales del Parto Natural, respecto a la atención médica habitual del parto, es que hemos revisado el impacto de cada una de las intervenciones involucradas en este. La atención del parto en escenarios alternativos a la clásica sala de parto hospitalaria, resultan seguros para la madre y el feto, y reducen significativamente las intervenciones médicas, por lo que parecen ser una opción razonable para aquellas mujeres que buscan el Parto Natural.

La operación cesárea se ha transformado en un problema a nivel mundial, por su elevada incidencia, justificada habitualmente por razones espurias, y no en la búsqueda de la mejor atención médica (Gibbons et al., 2010). En esta revisión hemos comprobado los beneficios maternos y perinatales del parto vaginal, lo que debe ser expuesto con claridad a las parejas desde el primer control prenatal (Spong, 2015). Entre las muchas razones propuestas para el aumento en la tasa de cesáreas, se encuentran las inducciones no justificadas en razones médicas; sin embargo, la veracidad de este acierto es puesta en duda por los datos de esta revisión, que muestran que la inducción rutinaria del trabajo de parto a las 39 semanas o más, no aumenta la incidencia de operación cesárea. Es posible concluir, en coincidencia con otras revisiones sobre el tema, que la relación entre la inducción y la tasa de operación cesárea puede ser debatida (Little \& Caughey, 2015).

El manejo activo del trabajo de parto reduce la duración de la fase activa sin mayores beneficios maternos o fetales; del mismo modo, la aceleración ocitócica y rotura artificial de membranas, de modo rutinario, no cambian la progresión del trabajo de parto. Con estos antecedentes, consideramos que la aceleración ocitócica y rotura artificial de membranas pueden ser evitadas en pacientes con trabajo de parto de progresión normal. Sin embargo, en aquellas pacientes con fase de dilatación prolongada, el uso de amniotomía y aceleración ocitócica ha demostrado una reducción en la tasa de cesárea, y deben ser implementados.

La analgesia epidural es altamente eficaz en la reducción del dolor asociado al parto, sin cambios en la tasa global de cesárea. 
Sin embargo, ha demostrado un aumento en el riesgo de parto vaginal asistido, y morbilidad materna leve; es por esto que debe ser ofrecida según los deseos de la paciente, comprendiendo beneficios y riesgos, pero sabiendo que no es imprescindible para el manejo y atención del parto.

El uso rutinario de la episiotomía apareció en la obstetricia moderna de la mano de la opinión de expertos, y no de buena evidencia científica. Sin embargo, ya desde fines del siglo pasado, se ha comprendido que la episiotomía restrictiva debería ser el estándar en la actualidad, pues al compararla con episiotomía rutinaria ha demostrado una reducción significativa de la morbilidad materna.

La monitorización de latidos cardíacos fetales es imprescindible durante el trabajo de parto, puesto que determina una importante reducción de la muerte perinatal (más de 20 veces). Consideramos que no es razonable acceder a la solicitud de una madre de no vigilar los latidos cardíacos fetales durante el trabajo de parto, pues se arriesga a graves complicaciones fetales, incluso la muerte. La vigilancia de los latidos fetales puede ser realizada de manera intermitente considerando una matrona por paciente y cumpliendo de manera estricta los tiempos de intervalo entre evaluación. Sin embargo para decidir entre auscultación intermitente o cardiotocografía continua, se debe considerar que la primera se asocia a menor tasa de cesáreas y parto vaginal operatorio, pero está relacionada con un incremento significativo en el riesgo de convulsiones neonatales. Esta información debe ser considerada por el equipo médico y la paciente al momento de decidir una opción de manejo.

La atención médica del parto ha sido uno de los grandes progresos de la humanidad, desde el acompañamiento por la partera hasta transformar la obstetricia en una especialidad médica básica, sin duda que las mujeres y sus hijos han sido mejor atendidos. Sin embargo, el exceso de celo en la acción médica ha llevado a la toma de acciones potencialmente innecesarias, lo que muchos han llamado"medicalización del parto" (Indraccolo et al., 2010; Weeks et al., 2016). Quienes defienden el Parto Natural, ven en la medicalización del parto una amenaza, haciendo razonable la expectativa del parto en el domicilio como un modo de evitar las intervenciones. La evidencia actual demuestra que el parto en el domicilio reduce las intervenciones médicas, pero a un riesgo demasiado alto, pues el parto en el domicilio se asocia a aumento en la tasa de muerte perinatal, convulsiones neonatales y necesidad de transfusiones sanguíneas maternas. Por ello, aquellos países que disponen de regulaciones al parto en el domicilio, han reservado esta opción solo a mujeres de bajo riesgo (Zielinski et al., 2015). Pensamos que reservar el parto en domicilio solo a mujeres de bajo riesgo es la mejor demostración de que no acudir al hospital para la atención del parto incrementa el riesgo de morbilidad materna y perinatal, pues si el riesgo fuera el mismo, entonces cualquier mujer podría optar a tener el parto en su casa. Disponer de escenarios alternativos para el parto dentro del hospital sí parece ser una opción segura para buscar lo mejor de ambos mundos.

Es importante analizar el concepto de "medicalización del parto", pues podría confundirse con el de atención médica del parto. Quienes usan el término "medicalización", sin duda lo hacen con afán despectivo, para desprestigiar a las intervenciones médicas. Sin embargo, la atención médica no consiste en efectuar todas las intervenciones posibles y disponibles por el solo hecho de aplicarlas, un buen acto médico se cimienta en la "medicina basada en evidencia", entendiendo por tal el uso de la mejor evidencia disponible, asociada al buen juicio clínico y a las preferencias del paciente para lograr la mejor atención médica posible (Kang, 2016). De este modo, la medicalización corresponde al uso irracional de las tecnologías médicas, y no al actuar médico correcto; entendiendo esto, obviamente la medicalización del parto es un acto nocivo para la salud de las personas. Por el contrario, el uso de intervenciones médicas útiles, racionalmente utilizadas y en concordancia con las preferencias de las pacientes, debe ser recomendado.

La participación de Doulas durante el trabajo de parto ha demostrado una disminución en el uso de analgesia, así como efectos psicológicos positivos en la madre, no existiendo ningún beneficio desde el punto de vista médico (materno o neonatal). Creemos necesario considerar esta información para evitar gastos innecesarios a las parejas; en Chile, las matronas desempeñan correctamente este rol de acompañamiento de las mujeres, y siendo profesionales muy bien preparados, sin duda evitarán riesgos innecesarios asociados a impedir el acceso de las mujeres a una atención segura del parto.

La posición vertical durante el parto es un deseo de muchas comunidades, y se sustenta además en bases biomecánicas muy razonables (Desseauve et al., 2017). En esta revisión hemos determinado que esta posición acorta el expulsivo en alrededor de 5 minutos, y se asocia a reducción en la necesidad de parto asistido, episiotomía y dolor. Sin embargo, la postura vertical durante el expulsivo se relaciona con mayor riesgo de desgarros perineales y de hemorragia posparto. Los beneficios y los problemas de no asumir la posición de litotomía para la atención del expulsivo deben ser discutidos con las mujeres, idealmente, antes de llegar al momento del parto.

La inmersión en el agua durante el trabajo de parto, parece ser reconfortante; su uso ha demostrado una reducción del uso de anestesia, sin otras diferencias con respecto al parto en posición supina o litotomía. No existen relatos históricos que muestren o 
sugieran que en algún momento de la historia de la humanidad el parto fue atendido en el agua, de modo que sostener que esta es una opción de Parto Natural es algo que debe debatirse.

El manejo activo del alumbramiento ha demostrado reducción significativa de la morbilidad materna, por lo que consideramos indispensable el incorporarlo a la práctica de rutina. Sin embargo, ante la evidencia actual del beneficio neonatal de la ligadura diferida del cordón umbilical (al menos un minuto), destacamos que al menos el uso de ocitocina posparto es indispensable en el manejo del alumbramiento para prevenir hemorragia posparto y reducir el riesgo de anemia.

El parto natural está tomando cada vez más fuerza en nuestro país, y es lícito preguntarse por las razones de esta tendencia. Si se debe a la decisión informada de las mujeres y los médicos de evitar intervenciones innecesarias o incluso optar por algunas que confieren un riesgo menor, parece ser una gran iniciativa. Pero si esta conducta es solo producto de estrategias de marketing de las clínicas o médicos para atraer "clientes", nos parece una situación alejada del buen acto médico. La decisión de optar por el Parto Natural debe ser discutida con las mujeres que lo solicitan, intentando aclarar con precisión cuál es su concepto del parto natural, explicándoles los riesgos y beneficios asociados a cada una de las intervenciones, y estableciendo de modo consensuado un plan de manejo (Lothian, 2000; MacDonald, 2011).

\section{Bibliografía}

Alfirevic Z, Devane D \& Gyte GM. (2006). Continuous cardiotocography (CTG) as a form of electronic fetal monitoring (EFM) for fetal assessment during labour. The Cochrane database of systematic reviews, CD006066.

Anim-Somuah M, Smyth RM \& Jones L. (2011). Epidural versus non-epidural or no analgesia in labour. The Cochrane database of systematic reviews, CD000331.

Austin MP \& Leader L. (2000). Maternal stress and obstetric and infant outcomes: epidemiological findings and neuroendocrine mechanisms. The Australian \& New Zealand journal of obstetrics \& gynaecology 40, 331-337.

Bager P, Wohlfahrt J \& Westergaard T. (2008). Caesarean delivery and risk of atopy and allergic disease: meta-analyses. Clinical and experimental allergy : journal of the British Society for Allergy and Clinical Immunology 38, 634-642.

Bauer ST \& Bonanno C. (2009). Abnormal placentation. Seminars in perinatology $33,88-96$.
Begley CM, Gyte GM, Devane D, McGuire W \& Weeks A. (2015). Active versus expectant management for women in the third stage of labour. The Cochrane database of systematic reviews, CD007412.

Bergstrom M, Kieler H \& Waldenstrom U. (2009). Effects of natural childbirth preparation versus standard antenatal education on epidural rates, experience of childbirth and parental stress in mothers and fathers: a randomised controlled multicentre trial. BJOG : an international journal of obstetrics and gynaecology 116, 1167-1176.

Birthplace in England Collaborative G, Brocklehurst P, Hardy P, Hollowell J, Linsell L, Macfarlane A, McCourt C, Marlow N, Miller A, Newburn M, Petrou S, Puddicombe D, Redshaw M, Rowe R, Sandall J, Silverton L \& Stewart M. (2011). Perinatal and maternal outcomes by planned place of birth for healthy women with low risk pregnancies: the Birthplace in England national prospective cohort study. Bmj 343, d7400.

Bloom SL, Casey BM, Schaffer JI, McIntire DD \& Leveno KJ. (2006). A randomized trial of coached versus uncoached maternal pushing during the second stage of labor. American journal of obstetrics and gynecology 194, 10-13.

Bulletins-Obstetrics ACoP. (2004). ACOG Practice Bulletin. Clinical management guidelines for obstetricians-gynecologists. Number 55, September 2004 (replaces practice pattern number 6, October 1997). Management of Postterm Pregnancy. Obstetrics and gynecology 104, 639-646.

Campbell D, Scott KD, Klaus MH \& Falk M. (2007). Female relatives or friends trained as labor doulas: outcomes at 6 to 8 weeks postpartum. Birth 34, 220-227.

Cardwell CR, Stene LC, Joner G, Cinek O, Svensson J, Goldacre MJ, Parslow RC, Pozzilli P, Brigis G, Stoyanov D, Urbonaite B, Sipetic S, Schober E, lonescu-Tirgoviste C, Devoti G, de Beaufort CE, Buschard K \& Patterson CC. (2008). Caesarean section is associated with an increased risk of childhood-onset type 1 diabetes mellitus: a meta-analysis of observational studies. Diabetologia 51, 726-735.

Carroli G \& Mignini L. (2009). Episiotomy for vaginal birth. The Cochrane database of systematic reviews, CD000081.

Cluett ER, Nikodem VC, McCandlish RE \& Burns EE. (2004). Immersion in water in pregnancy, labour and birth. The Cochrane database of systematic reviews, CD000111.

Crowley P. (2000). Interventions for preventing or improving the outcome of delivery at or beyond term. The Cochrane database of systematic reviews, CD000170. 
Chalmers B. (1992). WHO appropriate technology for birth revisited. British journal of obstetrics and gynaecology 99, 709-710.

Chez BF, Harvey MG \& Harvey CJ. (2000). Intrapartum fetal monitoring: past, present, and future. The Journal of perinatal \& neonatal nursing 14, 1-18.

Desseauve D, Fradet L, Lacouture P \& Pierre F. (2017). Position for labor and birth: State of knowledge and biomechanical perspectives. European journal of obstetrics, gynecology, and reproductive biology 208, 46-54.

Dietz HP \& Exton L. (2016). Response to 'Every CS must count'. The Australian \& New Zealand journal of obstetrics \& gynaecology 56, 556.

Eckert K, Turnbull D \& MacLennan A. (2001). Immersion in water in the first stage of labor: a randomized controlled trial. Birth 28, 84-93.

Gibbons L, Belizán JM, Lauer JA, Betrán AP, Merialdi M \& Althabe F. (2010). The global numbers and costs of additionally needed and unnecessary caesarean sections performed per year: overuse as a barrier to universal coverage. World Health Organization, http://www.who. int/healthsystems/topics/financing/healthreport/30Csectioncosts.pdf.

Gordon NP, Walton D, McAdam E, Derman J, Gallitero G \& Garrett L. (1999). Effects of providing hospital-based doulas in health maintenance organization hospitals. Obstetrics and gynecology 93, 422-426.

Gregory KD, Jackson S, Korst L \& Fridman M. (2012). Cesarean versus vaginal delivery: whose risks? Whose benefits? American journal of perinatology 29, 7-18.

Gupta JK \& Hofmeyr GJ. (2004). Position for women during second stage of labour. The Cochrane database of systematic reviews, CD002006.

Gupta JK, Hofmeyr GJ \& Shehmar M. (2012). Position in the second stage of labour for women without epidural anaesthesia. The Cochrane database of systematic reviews, CD002006.

Hodnett ED, Downe S \& Walsh D. (2012). Alternative versus conventional institutional settings for birth. The Cochrane database of systematic reviews, CD000012.

Indraccolo U, Calabrese S, Di lorio R, Corosu L, Marinoni E \& Indraccolo SR. (2010). Impact of the medicalization of labor on mode of delivery. Clinical and experimental obstetrics \& gynecology 37, 273-277.

Kagawa Singer M, Dressler W, George S \& Panel NIHE. (2016). Culture: The missing link in health research. Social science \& medicine 170, 237-246.
Kang H. (2016). How to understand and conduct evidence-based medicine. Korean journal of anesthesiology 69, 435-445.

Li Y, Tian Y, Zhu W, Gong J, Gu L, Zhang W, Guo Z, Li N \& Li J. (2014). Cesarean delivery and risk of inflammatory bowel disease: a systematic review and meta-analysis. Scandinavian journal of gastroenterology 49, 834-844.

Little SE \& Caughey AB. (2015). Induction of Labor and Cesarean: What is the True Relationship? Clinical obstetrics and gynecology 58, 269-281.

Lothian JA. (2000). Why natural childbirth? The Journal of perinatal education 9, 44-46.

MacDonald ME. (2011). The cultural evolution of natural birth. Lancet 378, 394-395.

MINSAL DCVDPyCdESdSP. (2008). Manual de Atención Personalizada en el Proceso Reproductivo. Trama Impresores S.A, Santiago, Chile.

Olsen O \& Clausen JA. (2012). Planned hospital birth versus planned home birth. The Cochrane database of systematic reviews, CD000352.

Pates JA \& Satin AJ. (2005). Active management of labor. Obstetrics and gynecology clinics of North America 32, 221-230, viii.

Saccone G \& Berghella V. (2015). Induction of labor at full term in uncomplicated singleton gestations: a systematic review and metaanalysis of randomized controlled trials. American journal of obstetrics and gynecology 213, 629-636.

Sanchez-Ramos L, Olivier F, Delke I \& Kaunitz AM. (2003). Labor induction versus expectant management for postterm pregnancies: a systematic review with meta-analysis. Obstetrics and gynecology 101, 1312-1318.

Smyth RM, Markham C \& Dowswell T. (2013). Amniotomy for shortening spontaneous labour. The Cochrane database of systematic reviews, CD006167.

Snowden JM, Tilden EL, Snyder J, Quigley B, Caughey AB \& Cheng YW. (2015). Planned Out-of-Hospital Birth and Birth Outcomes. The New England journal of medicine 373, 2642-2653.

Spong CY. (2015). Prevention of the first cesarean delivery. Obstetrics and gynecology clinics of North America 42, 377-380.

Thavagnanam S, Fleming J, Bromley A, Shields MD \& Cardwell CR. (2008). A meta-analysis of the association between Caesarean section and childhood asthma. Clinical and experimental allergy:journal of the British Society for Allergy and Clinical Immunology 38, 629-633. 
Carvajal et al.

Villar J, Valladares E, Wojdyla D, Zavaleta N, Carroli G, Velazco A, Shah A, Campodonico L, Bataglia V, Faundes A, Langer A, Narvaez A, Donner A, Romero M, Reynoso S, de Padua KS, Giordano D, Kublickas $M$, Acosta A, maternal WHOgso \& perinatal health research g. (2006). Caesarean delivery rates and pregnancy outcomes: the 2005 WHO global survey on maternal and perinatal health in Latin America. Lancet 367, 1819-1829.

Waldenstrom U \& Nilsson CA. (1993). Women's satisfaction with birth center care: a randomized, controlled study. Birth 20, 3-13.

Waldenstrom U \& Nilsson CA. (1994). Experience of childbirth in birth center care. A randomized controlled study. Acta obstetricia et gynecologica Scandinavica 73, 547-554.

Weeks F, Pantoja L, Ortiz J, Foster J, Cavada G \& Binfa L. (2016). Labor and Birth Care Satisfaction Associated With Medical Interventions and Accompaniment During Labor Among Chilean Women. Journal of midwifery \& women's health.
Wei S, Wo BL, Qi HP, Xu H, Luo ZC, Roy C \& Fraser WD. (2013). Early amniotomy and early oxytocin for prevention of, or therapy for, delay in first stage spontaneous labour compared with routine care. The Cochrane database of systematic reviews, CD006794.

Wei SQ, Luo ZC, Xu H \& Fraser WD. (2009). The effect of early oxytocin augmentation in labor: a meta-analysis. Obstetrics and gynecology 114, 641-649.

Westhoff G, Cotter AM \& Tolosa JE. (2013). Prophylactic oxytocin for the third stage of labour to prevent postpartum haemorrhage. The Cochrane database of systematic reviews, CD001808.

Zielinski R, Ackerson K \& Kane Low L. (2015). Planned home birth: benefits, risks, and opportunities. International journal of women's health 7, 361-377. 